\title{
Management of hyperglycaemia in type 2 diabetes, 2015: a patient-centred approach. Update to a Position Statement of the American Diabetes Association and the European Association for the Study of Diabetes
}

\author{
Silvio E. Inzucchi • Richard M. Bergenstal • John B. Buse • Michaela Diamant • \\ Ele Ferrannini • Michael Nauck • Anne L. Peters • Apostolos Tsapas • \\ Richard Wender • David R. Matthews
}

Received: 15 October 2014 / Accepted: 20 October 2014 / Published online: 13 January 2015

(C) Springer-Verlag Berlin Heidelberg 2015

Keywords Guidelines $\cdot$ Insulin $\cdot$ Oral agents $\cdot$ Therapy · Type

2 diabetes

\begin{abstract}
Abbreviations
CKD Chronic kidney disease

DPP-4 Dipeptidyl peptidase 4

eGFR Estimated GFR
\end{abstract}

Michaela Diamant is credited posthumously. Her experience, wisdom and wit were key factors in the creation of the original 2012 position statement; they continued to resonate with us during the writing of this update.

S. E. Inzucchi and D. R. Matthews were co-chairs for the Position Statement Writing Group. R. M. Bergenstal, J. B. Buse, A. L. Peters and R. Wender were the Writing Group for the American Diabetes Association. M. Diamant, E. Ferrannini, M. Nauck and A. Tsapas were the Writing Group for the European Association for the Study of Diabetes.

Simultaneous publication: This article is being simultaneously published in Diabetes Care and Diabetologia by the American Diabetes Association and the European Association for the Study of Diabetes. Copyright 2014 by the American Diabetes Association and SpringerVerlag. Copying with attribution allowed for any non-commercial use of the work.

Electronic supplementary material The online version of this article (doi:10.1007/s00125-014-3460-0) contains an ESM slide set for this paper and an abridged version, which is available to authorised users.

S. E. Inzucchi

Section of Endocrinology, Yale University School of Medicine,

Yale-New Haven Hospital, New Haven, CT, USA

R. M. Bergenstal International Diabetes Center at Park Nicollet, Minneapolis, MN, USA

GLP-1 Glucagon-like peptide 1

SGLT2 Sodium-glucose co-transporter 2

TZD Thiazolidinedione

In 2012, the American Diabetes Association (ADA) and the European Association for the Study of Diabetes (EASD) published a position statement on the

J. B. Buse

Division of Endocrinology, University of North Carolina School of Medicine, Chapel Hill, NC, USA

M. Diamant

Diabetes Center/Department of Internal Medicine, VU University

Medical Center, Amsterdam, The Netherlands 
management of hyperglycaemia in patients with type 2 diabetes $[1,2]$. This was needed because of an increasing array of anti-hyperglycaemic drugs and growing uncertainty regarding their proper selection and sequence. Because of a paucity of comparative effectiveness research on long-term treatment outcomes with many of these medications, the 2012 publication was less prescriptive than prior consensus reports. We previously described the need to individualise both treatment targets and treatment strategies, with an emphasis on patient-centred care and shared decision-making, and this continues to be our position, although there are now more head-to-head trials that show slight variance between agents with regard to glucose-lowering effects. Nevertheless, these differences are often small and would be unlikely to reflect any definite differential effect in an individual patient.

The ADA and EASD have requested an update to the position statement incorporating new data from recent clinical trials. Between June and September of 2014, the Writing Group reconvened, including one face-to-face meeting, to discuss the changes. An entirely new statement was felt to

E. Ferrannini

Department of Medicine, University of Pisa School of Medicine, Pisa, Italy

M. Nauck

Diabeteszentrum Bad Lauterberg, Bad Lauterberg im Harz, Germany

A. L. Peters

Division of Endocrinology, Keck School of Medicine of the

University of Southern California, Los Angeles, CA, USA

\section{A. Tsapas}

Second Medical Department, Aristotle University Thessaloniki, Thessaloniki, Greece

R. Wender

American Cancer Society, Atlanta, GA, USA

R. Wender

Department of Family and Community Medicine, Jefferson Medical College, Thomas Jefferson University, Philadelphia, PA, USA

D. R. Matthews $(\triangle)$

Harris Manchester College (University of Oxford),

Mansfield Road, Oxford OX1 3TD, UK

e-mail: david.matthews@ocdem.ox.ac.uk

\section{R. Matthews}

Oxford Centre for Diabetes, Endocrinology and Metabolism, Churchill Hospital, Oxford, UK

D. R. Matthews

National Institute for Health Research (NIHR), Oxford Biomedical Research Centre, Oxford, UK be unnecessary. Instead, the group focused on those areas where revisions were suggested by a changing evidence base. This briefer article should therefore be read as an addendum to the previous full account $[1,2]$.

\section{Glycaemic targets}

Glucose control remains a major focus in the management of patients with type 2 diabetes. However, this should always be in the context of a comprehensive cardiovascular risk factor reduction programme, to include smoking cessation and the adoption of other healthy lifestyle habits, blood pressure control, lipid management with priority to statin medications and, in some circumstances, antiplatelet therapy. Studies have conclusively determined that reducing hyperglycaemia decreases the onset and progression of microvascular complications [3, 4]. The impact of glucose control on cardiovascular complications remains uncertain; a more modest benefit is likely to be present, but probably emerges only after many years of improved control [5]. Results from large trials have also suggested that overly aggressive control in older patients with more advanced disease may not have significant benefits and may indeed present some risk [6]. Accordingly, instead of a one-size-fits-all approach, personalisation is necessary, balancing the benefits of glycaemic control with its potential risks, taking into account the adverse effects of glucoselowering medications (particularly hypoglycaemia), and the patient's age and health status, among other concerns. Figure 1 displays those patient and disease factors that may influence the target for glucose control, as reflected by $\mathrm{HbA}_{1 \mathrm{c}}$. The main update to this figure is the separation of those factors that are potentially modifiable from those that are usually not. The patient's attitude and expected treatment efforts and access to resources and support systems are unique in so far as they may improve (or worsen) over time. Indeed, the clinical team should encourage patient adherence to therapy through education and also try to optimise care in the context of prevailing health coverage and/or the patient's financial means. Other features, such as age, life expectancy, comorbidities and the risks and consequences to the patient from an adverse drug event, are more or less fixed. Finally, the usual $\mathrm{HbA}_{1 \mathrm{c}}$ goal cut-off point of $7 \%(53.0 \mathrm{mmol} / \mathrm{mol})$ has also been inserted at the top of the figure to provide some context to the recommendations regarding stringency of treatment efforts.

Therapeutic options (See text box 'Properties of available glucose-lowering agents in the USA and Europe that may guide individualised treatment choices in patients with type 2 diabetes'; for other unchanged options, also refer to the original statement $[1,2])$ 


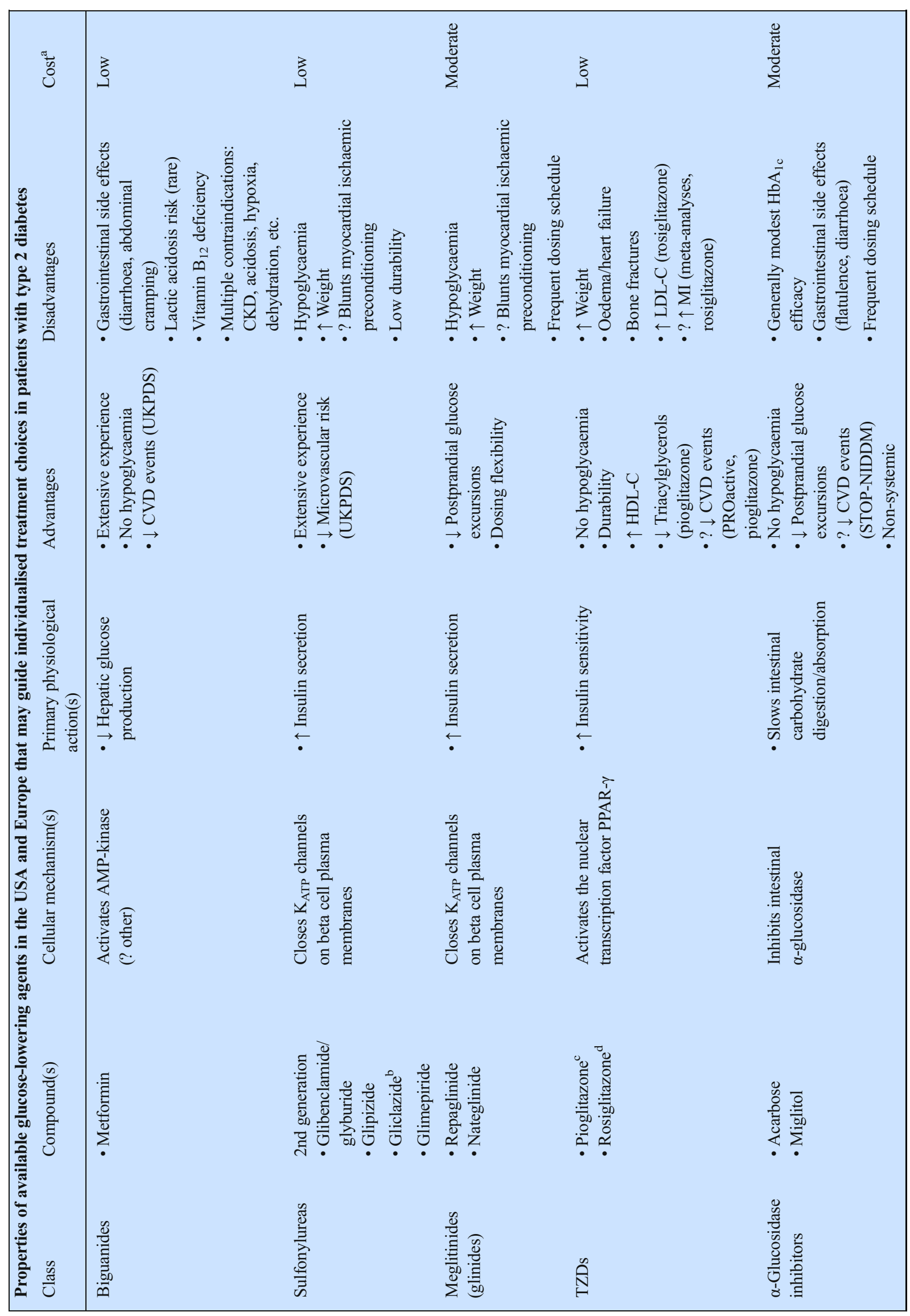


홍

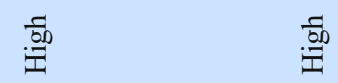

$\stackrel{50}{.00}$

.5몽

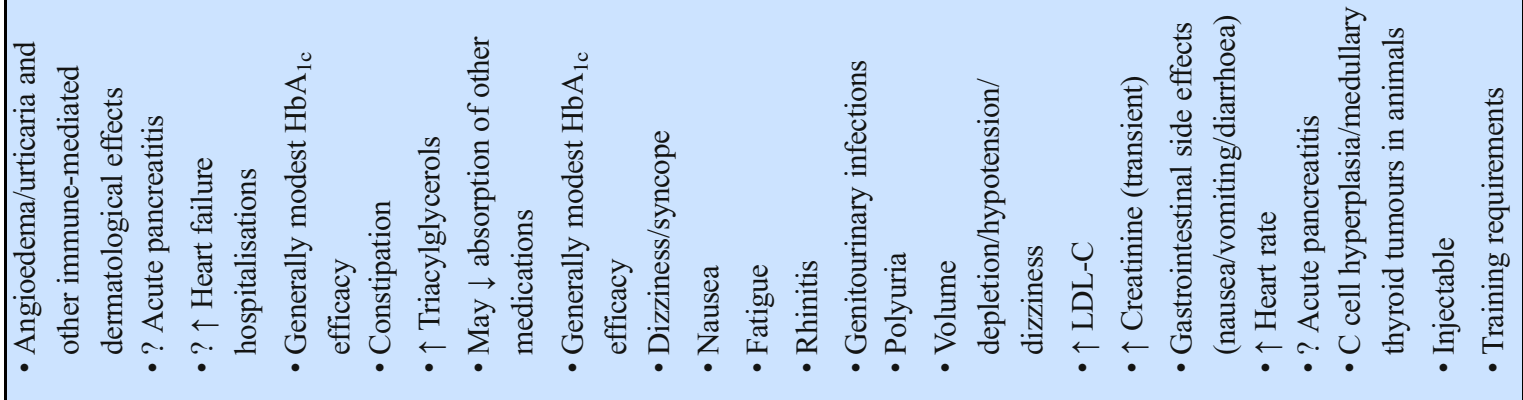

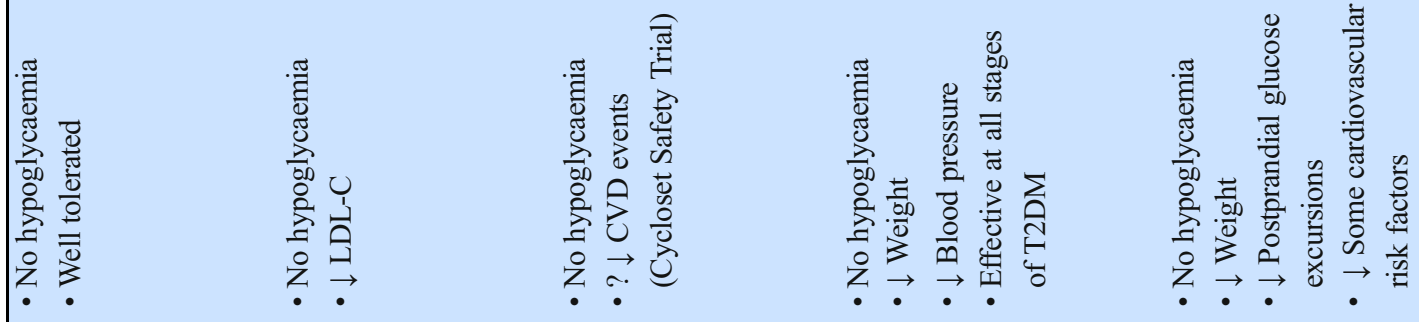

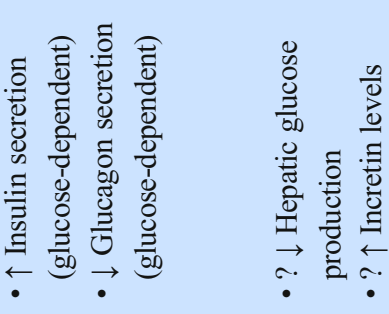

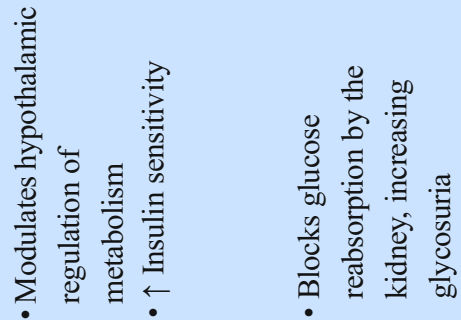

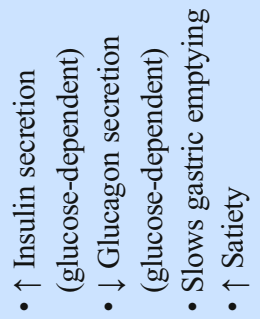

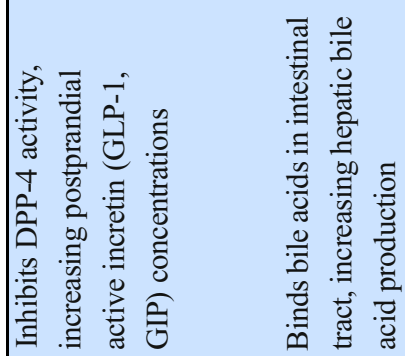
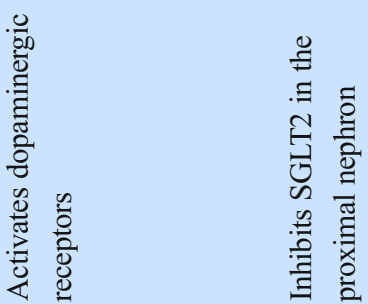

苋

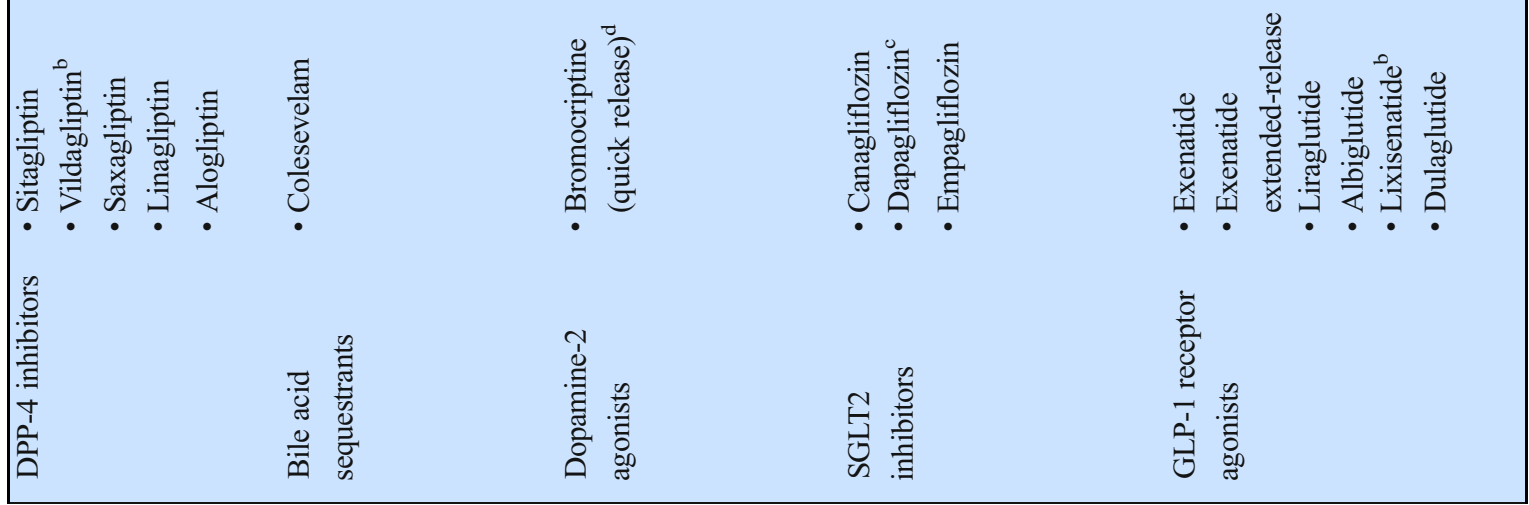




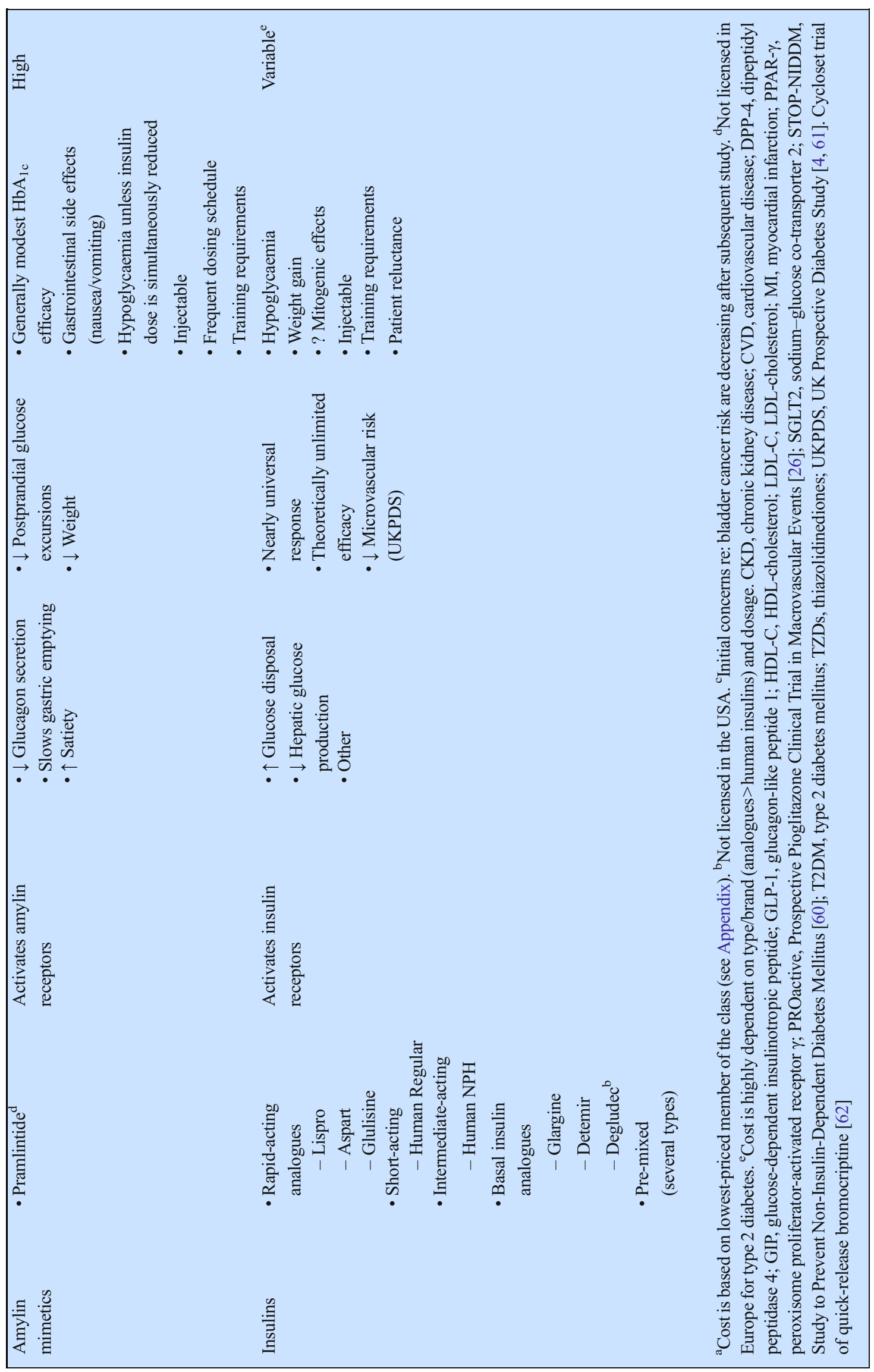




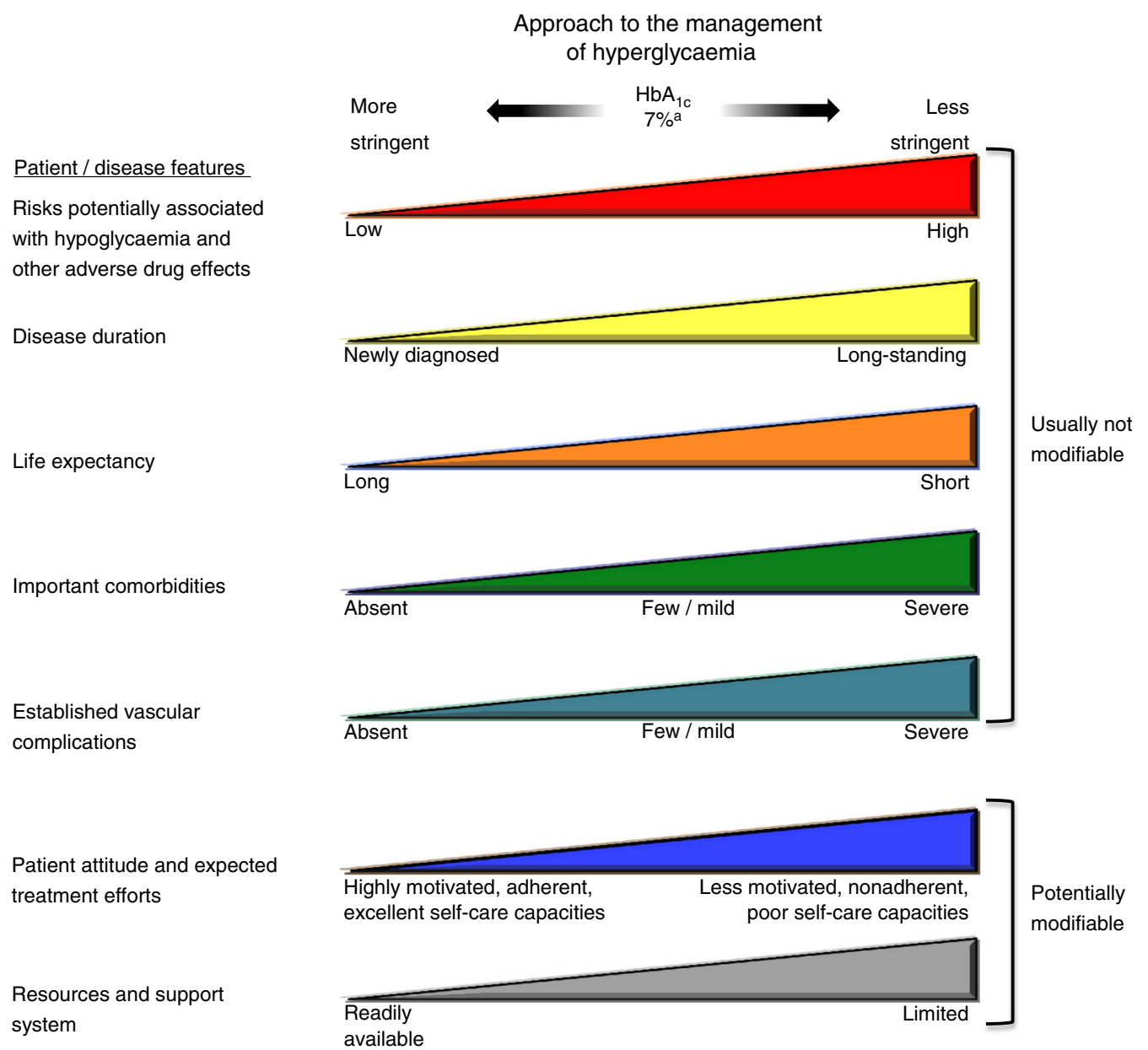

Fig. 1 Modulation of the intensiveness of glucose lowering in type 2 diabetes. Depiction of patient and disease factors that may be used by the practitioner to determine optimal $\mathrm{HbA}_{1 \mathrm{c}}$ targets in patients with type 2 diabetes. Greater concerns regarding a particular domain are represented by increasing height of the corresponding ramp. Thus, characteristics/ predicaments toward the left justify more stringent efforts to lower

Sodium-glucose co-transporter 2 inhibitors The major change in treatment options since the publication of the 2012 position statement has been the availability of a new class of glucose-lowering drugs, the sodium-glucose co-transporter 2 (SGLT2) inhibitors [7]. These agents reduce $\mathrm{HbA}_{1 \mathrm{c}}$ by $0.5-1.0 \%(5.5-11 \mathrm{mmol} / \mathrm{mol})$ vs placebo $[7,8]$. When compared with most standard oral agents in head-to-head trials, they appear to be roughly similarly efficacious with regard to initial $\mathrm{HbA}_{1 \mathrm{c}}$ lowering [9-12]. Their mechanism of action involves inhibiting the SGLT2 in the proximal nephron, thereby reducing glucose reabsorption and increasing urinary glucose excretion by up to $80 \mathrm{~g} /$ day $[13,14]$. Because this action is independent of insulin, SGLT2 inhibitors may be used at any stage of type 2 diabetes, even after insulin secretion has waned significantly. Additional potential advantages include modest weight loss $(\sim 2 \mathrm{~kg}$, stabilising over 6 12 months) and consistent lowering of systolic and diastolic
$\mathrm{HbA}_{1 \mathrm{c}}$, whereas those toward the right suggest (indeed, sometimes mandate) less stringent efforts. Where possible, such decisions should be made with the patient, reflecting his or her preferences, needs and values. This 'scale' is not designed to be applied rigidly but to be used as a broad construct to guide clinical decision-making. Based on an original figure by Ismail-Beigi et al [59]. ${ }^{\mathrm{a}} \mathrm{HbA}_{1 \mathrm{c}} 7 \%=53 \mathrm{mmol} / \mathrm{mol}$

blood pressure in the order of $\sim 2-4 / \sim 1-2 \mathrm{mmHg}[7,8,15]$. Their use is also associated with reductions in plasma uric acid levels and albuminuria [16], although the clinical impact of these changes over time is unknown.

Side effects of SGLT2 inhibitor therapy include genital mycotic infections, at rates of about $11 \%$ higher in women and about $4 \%$ higher in men compared with placebo [17]; in some studies, a slight increase in urinary tract infections was shown $[7,9,12,17,18]$. They also possess a diuretic effect, and so symptoms related to volume depletion may occur [7, 19]. Consequently, these agents should be used cautiously in the elderly, in any patient already on a diuretic, and in anyone with a tenuous intravascular volume status. Reversible small increases in serum creatinine occur [14, 19]. Increased urine calcium excretion has been observed [20], and the United States Food and Drug Administration (FDA) mandated a follow-up of upper limb fractures of patients on canagliflozin 
after an adverse imbalance in cases was reported in short-term trials [21]. Small increases in LDL-cholesterol $(\sim 5 \%)$ have been noted in some trials, the implications of which are unknown. Due to their mechanism of action, SGLT2 inhibitors are less effective when the estimated GFR (eGFR) is $<45$ $60 \mathrm{ml} \mathrm{min}{ }^{-1}(1.73 \mathrm{~m})^{-2}$; currently available agents have variable label restrictions for values below this threshold.

Data on microvascular outcomes with SGLT2 inhibitors are lacking (as with most agents other than sulfonylureas and insulin). Effects on macrovascular disease are also unknown; cardiovascular safety trials are currently in progress [22].

Thiazolidinediones Earlier concerns that the thiazolidinediones (TZDs) - in particular pioglitazone - are associated with bladder cancer have largely been allayed by subsequent evidence [23-25]. These agents tend to cause weight gain and peripheral oedema and have been shown to increase the incidence of heart failure [26]. They also increase the risk of bone fractures, predominately in women [27]. Pioglitazone is now available as a generic drug, substantially decreasing its cost.

Dipeptidyl peptidase 4 inhibitors One large trial involving the dipeptidyl peptidase 4 (DPP-4) inhibitor saxagliptin found no overall cardiovascular risk or benefit (although the follow-up was only slightly more than 2 years) compared with placebo [28]. However, more heart failure hospitalisations occurred in the active therapy group $(3.5 \%$ vs $2.8 \%, p=0.007)[28,29]$. Alogliptin, another DPP-4 inhibitor, also did not have any demonstrable cardiovascular excess risk over an even shorter period (18 months) in high-risk patients [30]. A wider database interrogation indicated no signal for cardiovascular disease or heart failure [30,31]. Several other trials are underway, and until the results of these are reported, this class should probably be used cautiously, if at all, in patients with preexisting heart failure.

One area of concern with this class, as well as the other incretin-based category, the glucagon-like peptide 1 (GLP-1) receptor agonists, has been pancreatic safety — both regarding possible pancreatitis and pancreatic neoplasia. The prescribing guidelines for these drugs include cautions about using them in individuals with a prior history of pancreatitis. While this is reasonable, emerging data from large observational datasets [32], as well as from two large cardiovascular trials with DPP4 inhibitors [28-30], have found no statistically increased rates of pancreatic disease.

Generally speaking, the use of any drug in patients with type 2 diabetes must balance the glucose-lowering efficacy, side-effect profiles, anticipation of additional benefits, cost, and other practical aspects of care, such as dosing schedule and requirements for glucose monitoring. The patient-who is obviously the individual most affected by drug choice-should participate in a shared decision-making process regarding both the intensiveness of blood glucose control and which medications are to be selected.

\section{Implementation strategies}

Initial drug therapy (see Fig. 2) Metformin remains the optimal drug for monotherapy. Its low cost, proven safety record, weight neutrality and possible benefits on cardiovascular outcomes have secured its place as the favoured initial drug choice. There is increasing evidence that the current cut-off points for renal safety in the USA (contraindicated if serum creatinine $\geq 133 \mu \mathrm{mol} / 1[\geq 1.5 \mathrm{mg} / \mathrm{dl}]$ in men or $124 \mu \mathrm{mol} / 1$ $[1.4 \mathrm{mg} / \mathrm{dl}]$ in women) may be overly restrictive [33]. Accordingly, there are calls to relax prescribing polices to extend the use of this important medication to those with mild-moderate, but stable, chronic kidney disease (CKD) [34-36]. Many practitioners would continue to prescribe metformin even when the eGFR falls to less than $45-60 \mathrm{ml} \mathrm{m^{-1 }}(1.73 \mathrm{~m})^{-2}$, perhaps with dose adjustments to account for reduced renal clearance of the compound. One criterion for stopping the drug is an eGFR of $<30 \mathrm{ml} \mathrm{min}^{-1}(1.73 \mathrm{~m})^{-2}[34,37,38]$. Of course, any use in patients with CKD mandates diligent follow-up of renal function.

In circumstances where metformin is contraindicated or not tolerated, one of the second-line agents (see below) may be used, although the choices become more limited if renal insufficiency is the reason metformin is being avoided. In these circumstances it is unwise to use sulfonylureas, particularly glibenclamide (known as glyburide in the USA and Canada), because of the risk of hypoglycaemia. DPP-4 inhibitors are probably a preferable choice, although, with the exception of linagliptin [39], dosage adjustments are required.

Advancing to dual combination and triple combination therapy (see Fig. 2) While the SGLT2 inhibitors are approved as monotherapy, they are mainly used in combination with metformin and/or other agents [19]. Given their demonstrated efficacy and clinical experience to date, they are reasonable options as second-line or third-line agents [40-42] (Fig. 2). Similar to most combinations, efficacy may be less than additive when SGLT2 inhibitors are used in combination with DPP-4 inhibitors [43]. There are no data available on the use of SGLT2 inhibitors in conjunction with GLP-1 receptor agonists; an evidence-based recommendation for this combination cannot be made at this time.

As noted in the original position statement, initial combination therapy with metformin plus a second agent may allow patients to achieve $\mathrm{HbA}_{1 \mathrm{c}}$ targets more quickly than sequential therapy. Accordingly, such an approach may be considered in 


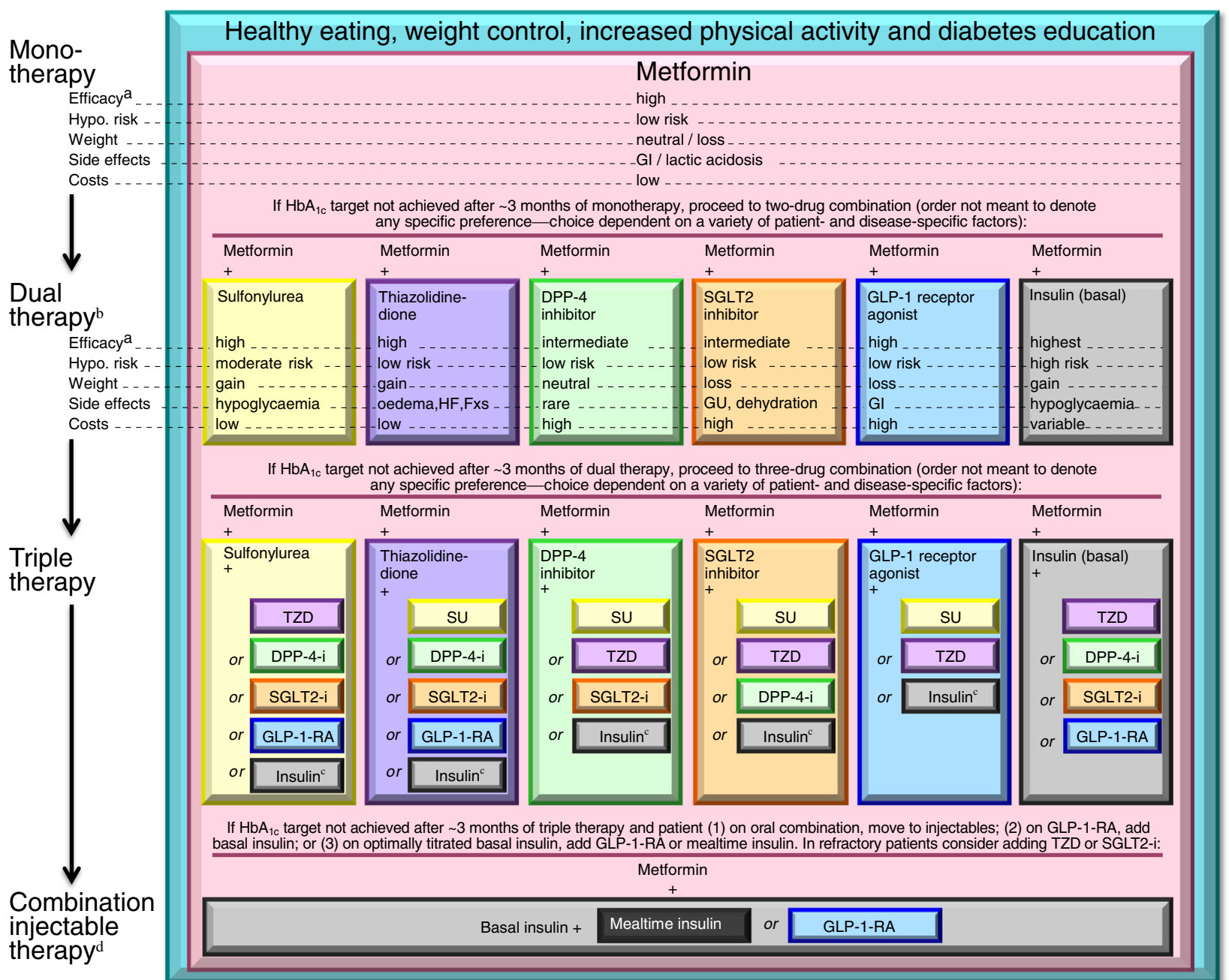

Fig. 2 Anti-hyperglycaemic therapy in type 2 diabetes: general recommendations. Potential sequences of anti-hyperglycaemic therapy for patients with type 2 diabetes are displayed, the usual transition being vertical, from top to bottom (although horizontal movement within therapy stages is also possible, depending on the circumstances). In most patients, begin with lifestyle changes; metformin monotherapy is added at, or soon after, diagnosis, unless there are contraindications. If the $\mathrm{HbA}_{1 \mathrm{c}}$ target is not achieved after $\sim 3$ months, consider one of the six treatment options combined with metformin: a sulfonylurea, TZD, DPP-4 inhibitor, SGLT2 inhibitor, GLP-1 receptor agonist or basal insulin. (The order in the chart, not meant to denote any specific preference, was determined by the historical availability of the class and route of administration, with injectables to the right and insulin to the far right.) Drug choice is based on patient preferences as well as various patient, disease and drug characteristics, with the goal being to reduce glucose concentrations while minimising side effects, especially hypoglycaemia. The figure emphasises drugs in common use in the USA and/or Europe. Rapid-acting secretagogues (meglitinides) may be used in place of sulfonylureas in patients with irregular meal schedules or who develop late postprandial hypoglycaemia on a sulfonylurea. Other drugs not shown ( $\alpha$-glucosidase inhibitors, colesevelam, bromocriptine, pramlintide) may be tried in specific situations (where available), but are generally not favoured because of their modest efficacy, the frequency of administration and/or limiting side effects. In patients intolerant of, or with contraindications for, metformin, consider initial drug from other classes depicted under 'Dual therapy' and proceed accordingly. In this circumstance, while published trials are generally lacking, it is reasonable to consider three-drug combinations that do not include metformin. Consider initiating therapy with a dual combination when $\mathrm{HbA}_{1 \mathrm{c}}$ is $\geq 9 \%$ ( $\geq 75 \mathrm{mmol} / \mathrm{mol}$ ) to more expeditiously achieve target. Insulin has the advantage of being effective where other agents may not be and should be considered a part of any combination regimen when hyperglycaemia is severe, especially if the patient is symptomatic or if any catabolic features (weight loss, any ketosis) are evident. Consider initiating combination injectable therapy with insulin when blood glucose is $\geq 16.7-19.4 \mathrm{mmol} / 1$ ( $\geq 300-350 \mathrm{mg} / \mathrm{dl}$ ) and $/$ or $\mathrm{HbA}_{1 \mathrm{c}} \geq 10-12 \%$ ( $\left.\geq 86-108 \mathrm{mmol} / \mathrm{mol}\right)$. Potentially, as the patient's glucose toxicity resolves, the regimen can be subsequently simplified. a See Appendix for description of efficacy categorisation. ${ }^{\mathrm{b}}$ Consider initial therapy at this stage when $\mathrm{HbA}_{\mathrm{cc}} \geq 9 \%$ ( $\geq 75 \mathrm{mmol} / \mathrm{mol}$ ). ${ }^{\mathrm{c}}$ Usually a basal insulin (e.g. NPH, glargine, [A21Gly,B31 Arg,B32Arg human insulin] detemir [B29Lys $(\varepsilon-$ tetradecanoyl),desB30 human insulin], degludec [des(B30)LysB29 $(\gamma-$ Glu Ne-hexadecandioyl) human insulin]). ${ }^{\mathrm{d}}$ Consider initial therapy at this stage when blood glucose is $\geq 16.7-19.4 \mathrm{mmol} / 1(\geq 300-350 \mathrm{mg} / \mathrm{dl})$ and/or $\mathrm{HbA}_{1 \mathrm{c}} \geq 10-12 \%$ ( $\left.\geq 86-108 \mathrm{mmol} / \mathrm{mol}\right)$, especially if patient is symptomatic or if catabolic features (weight loss, ketosis) are present, in which case basal insulin+mealtime insulin is the preferred initial regimen. DPP-4-i, DPP-4 inhibitor; Fxs, fractures; GI, gastrointestinal; GLP-1-RA, GLP-1 receptor agonist; GU, genito-urinary infections; HF, heart failure; hypo., hypoglycaemia; SGLT2-i, SGLT2 inhibitor; SU, sulfonylurea 
those individuals with baseline $\mathrm{HbA}_{1 \mathrm{c}}$ levels well above target, who are unlikely to successfully attain their goal using monotherapy. A reasonable threshold $\mathrm{HbA}_{1 \mathrm{c}}$ for this consideration is $\geq 9 \%(\geq 75 \mathrm{mmol} / \mathrm{mol})$. Of course, there is no proven overall advantage to achieving a glycaemic target more quickly by a matter of weeks or even months. Accordingly, as long as close patient follow-up can be ensured, prompt sequential therapy is a reasonable alternative, even in those with baseline $\mathrm{HbA}_{1 \mathrm{c}}$ levels in this range.

Combination injectable therapy (see Figs 2 and 3) In certain patients, glucose control remains poor despite the use of three anti-hyperglycaemic drugs in combination. With long-standing diabetes, a significant diminution in pancreatic insulin secretory capacity dominates the clinical picture. In any patient not achieving an agreed $\mathrm{HbA}_{1 \mathrm{c}}$ target despite intensive therapy, basal insulin should be considered an essential component of the treatment strategy. After basal insulin (usually in combination with metformin and sometimes an additional agent), the 2012 position statement endorsed the addition of one to three injections of a rapid-acting insulin analogue dosed before meals. As an alternative, the statement mentioned that, in selected patients, simpler (but somewhat less flexible) premixed formulations of intermediate- and short/rapid-acting insulins in fixed ratios could also be considered [44]. Over the past 3 years, however, the effectiveness of combining GLP-1 receptor agonists (both shorter-acting and newer weekly formulations) with basal insulin has been demonstrated, with most studies showing equal or slightly superior efficacy to the addition of prandial insulin, and with weight loss and less hypoglycaemia [45-47]. The available data now suggest that either a GLP-1 receptor agonist or prandial insulin could be used in this setting, with the former arguably safer, at least for short-term outcomes [45, 48, 49]. Accordingly, in those patients on basal insulin with one or more oral agents whose diabetes remains uncontrolled, the addition of a GLP-1 receptor agonist or mealtime insulin could be viewed as a logical progression of the treatment regimen, the former perhaps a more attractive option in more obese individuals or in those who may not have the capacity to handle the complexities of a multi-dose insulin regimen. Indeed, there is increasing evidence for and interest in this approach [50]. In those patients who do not respond adequately to the addition of a GLP-1 receptor agonist to basal insulin, mealtime insulin in a combined 'basal-bolus' strategy should be used instead [51].

In selected patients at this stage of disease, the addition of an SGLT2 inhibitor may further improve control and reduce the amount of insulin required [52]. This is particularly an issue when large doses of insulin are required in obese, highly insulin-resistant patients. Another, older, option, the addition of a TZD (usually pioglitazone), also has an insulin-sparing effect and may also reduce $\mathrm{HbA}_{1 \mathrm{c}}[53$, 54], but at the expense of weight gain, fluid retention and increased risk of heart failure. So, if used at this stage, low doses are advisable and only with very careful monitoring of the patient.

Concentrated insulins (e.g. U-500 Regular) also have a role in those individuals requiring very large doses of insulin per day, in order to minimise injection volume [55]. However, these must be carefully prescribed, with meticulous communication with both patient and pharmacist regarding proper dosing instructions.

Practitioners should also consider the significant expense and additional complexity and costs of multiple combinations of glucose-lowering medications. Overly burdensome regimens should be avoided. The inability to achieve glycaemic targets with an increasingly convoluted regimen should prompt a pragmatic reassessment of the $\mathrm{HbA}_{1 \mathrm{c}}$ target or, in the very obese, consideration of non-pharmacological interventions, such as bariatric surgery.

Of course, nutritional counselling and diabetes selfmanagement education are integral parts of any therapeutic programme throughout the disease course. These will ensure that the patient has access to information on methods to reduce, where possible, the requirements for pharmacotherapy, as well as to safely monitor and control blood glucose levels.

Clinicians should also be wary of the patient with latent autoimmune diabetes of adulthood (LADA), which may be identified by measuring islet antibodies, such as those against GAD65 [56]. Although control with oral agents is possible for a variable period of time, these individuals, who are typically but not always lean, develop insulin requirements faster than those with typical type 2 diabetes [57] and progressively manifest metabolic changes similar to those seen in type 1 diabetes. Ultimately, they are optimally treated with a regimen consisting of multiple daily injections of insulin, ideally using a basal-bolus approach (or an insulin pump).

Figure 3 has been updated to include proposed dosing instructions for the various insulin strategies, including the addition of rapid-acting insulin analogues before meals or the use of pre-mixed insulin formulations.

\section{Other considerations}

As emphasised in the original position statement, optimal treatment of type 2 diabetes must take into account the various comorbidities that are frequently encountered in patients, particularly as they age. These include coronary artery disease, heart failure, renal and liver disease, dementia and increasing propensity to (and greater likelihood of experiencing untoward outcomes from) hypoglycaemia. There are few new data to further this discussion. As mentioned, new concerns about 


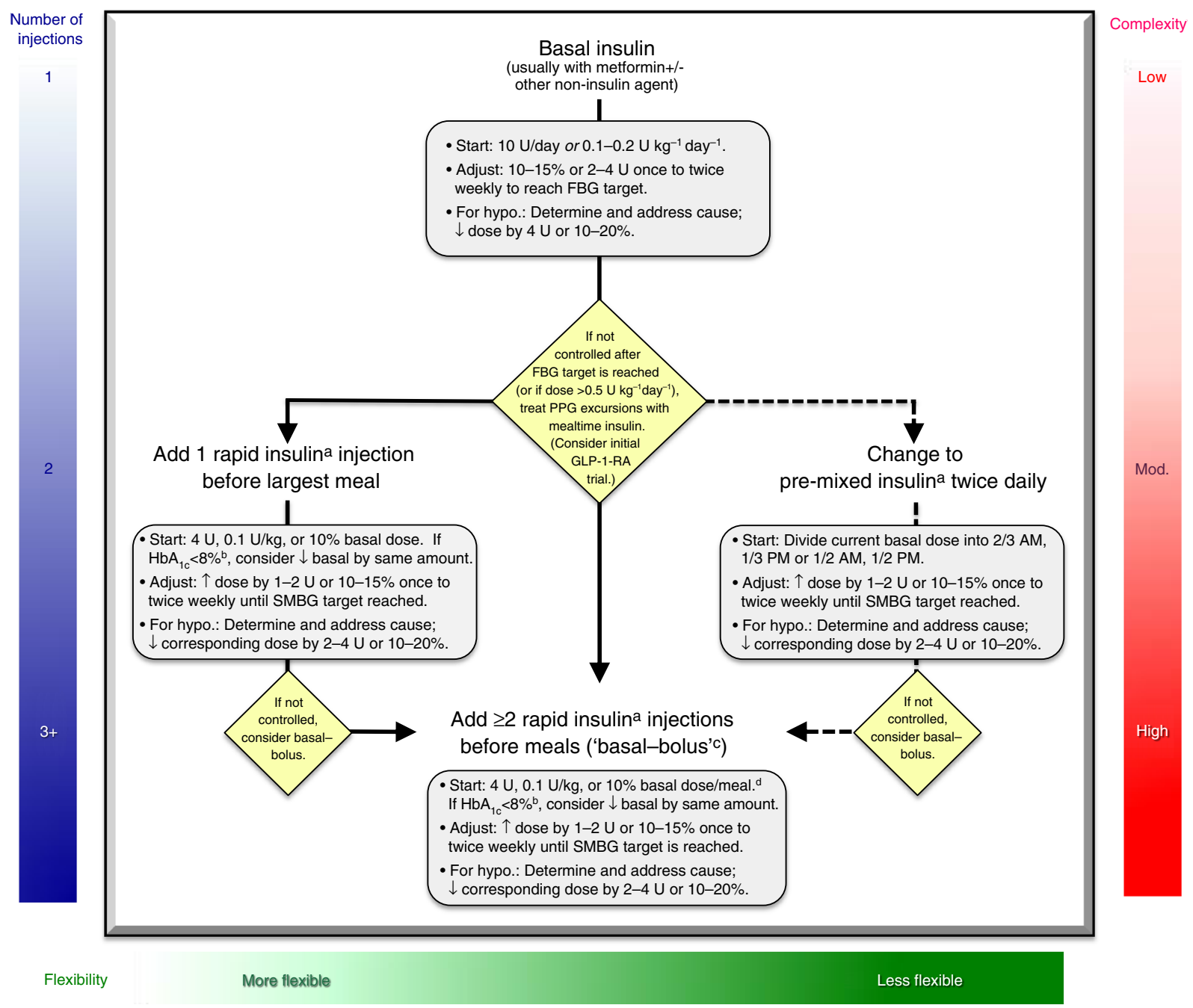

Fig. 3 Approach to starting and adjusting insulin in type 2 diabetes. This figure focuses mainly on sequential insulin strategies, describing the number of injections and the relative complexity and flexibility of each stage. Basal insulin alone is the most convenient initial regimen, beginning at $10 \mathrm{U}$ or $0.1-0.2 \mathrm{U} / \mathrm{kg}$, depending on the degree of hyperglycaemia. It is usually prescribed in conjunction with metformin and possibly one additional non-insulin agent. When basal insulin has been titrated to an acceptable fasting blood glucose but $\mathrm{HbA}_{1 \mathrm{c}}$ remains above target, consider proceeding to 'Combination injectable therapy' (see Fig. 2) to cover postprandial glucose excursions. Options include adding a GLP-1-RA (not shown) or a mealtime insulin, consisting of one to three injections of a rapid-acting insulin analogue ${ }^{\mathrm{a}}$ (lispro [B28Lys,B29Pro human insulin], aspart [B28Asp human insulin] or glulisine [B3Lys,B29Glu human insulin]) administered just before eating. A less studied alternative, transitioning from basal insulin to a twice daily pre-mixed (or biphasic) insulin analogue ${ }^{\mathrm{a}}$ (70/30 aspart mix, $75 / 25$ or 50/ 50 lispro mix), could also be considered. Once any insulin regimen is initiated, dose titration is important, with adjustments made in both mealtime and basal insulins based on the prevailing blood glucose levels, with knowledge of the pharmacodynamic profile of each formulation used (pattern control). Non-insulin agents may be continued, although sulfonylureas, DPP-4 inhibitors and GLP-1-RAs are typically stopped once insulin regimens more complex than basal are utilised. In refractory patients, however, especially in those requiring escalating insulin doses, adjunctive therapy with metformin and a TZD (usually pioglitazone) or SGLT2 inhibitor may be helpful in improving control and reducing the amount of insulin needed. Comprehensive education regarding SMBG, diet and exercise and the avoidance of, and response to, hypoglycaemia are critically important in any insulin-treated patient. FBG, fasting blood glucose; GLP-1-RA, GLP-1 receptor agonist; hypo., hypoglycaemia; Mod., moderate; PPG, postprandial glucose; SMBG, self-monitoring of blood glucose.

${ }^{\mathrm{a}}$ Regular human insulin and human NPH-Regular pre-mixed formulations (70/30) are less costly alternatives to rapid-acting insulin analogues and pre-mixed insulin analogues, but their pharmacodynamic profiles make them suboptimal for the coverage of postprandial glucose excursions. ${ }^{\mathrm{b}} \mathrm{HbA}_{1 \mathrm{c}} 8 \%=64 \mathrm{mmol} / \mathrm{mol} .{ }^{\mathrm{c}} \mathrm{A}$ less commonly used and more costly alternative to basal-bolus therapy with multiple daily injections in type 2 diabetes is continuous subcutaneous insulin infusion (insulin pump). ${ }^{\mathrm{d}}$ In addition to the suggestions provided for determining the starting dose of mealtime insulin under 'basal-bolus', another method consists of adding up the total current daily insulin dose and then providing one-half of this amount as basal and one-half as mealtime insulin, the latter split evenly between three meals
DPP-4 inhibitors and heart failure and the issues concerning SGLT2 inhibitors and renal status should be taken into consideration [29]. Finally, cost can be an important consideration in drug selection. As the 
prices of newer medications continue to increase, practitioners should take into account patient (and societal) resources and determine when less costly, generic products might be appropriately used.

\section{Future directions}

More long-term data regarding the cardiovascular impact of our glucose-lowering therapies will be available over the next 1-3 years. Information from these trials will further assist us in optimising treatment strategies. A large comparative effectiveness study in the USA is now assessing long-term outcomes with multiple agents after metformin monotherapy, but results are not anticipated until at least 2020 [58].

The recommendations in this position statement will obviously need to be updated in future years in order to provide the best and most evidence-based recommendations for patients with type 2 diabetes.

Acknowledgements This position statement was written by joint request of the ADA and the EASD Executive Committees, which have approved the final document. The process involved wide literature review, one face-to-face meeting of the Writing Group and multiple revisions via e-mail communications. We gratefully acknowledge the following experts who provided critical review of a draft of this update: James Best, Lee Kong Chian School of Medicine, Singapore; Henk Bilo, Isala Clinics, Zwolle, the Netherlands; Andrew Boulton, Manchester University, Manchester, UK; Paul Callaway, University of Kansas School of MedicineWichita, Wichita, KS, USA; Bernard Charbonnel, University of Nantes, Nantes, France; Stephen Colagiuri, The University of Sydney, Sydney, NSW, Australia; Leszek Czupryniak, Medical University of Lodz, Lodz, Poland; Margo Farber, University of Michigan Health System and College of Pharmacy, Ann Arbor, MI, USA; Richard Grant, Kaiser Permanente Northern California, Oakland, CA, USA; Faramarz Ismail-Beigi, Case Western Reserve University School of Medicine/Cleveland VA Medical Center, Cleveland, OH, USA; Darren McGuire, University of Texas Southwestern Medical Center, Dallas, TX, USA; Julio Rosenstock, Dallas Diabetes and Endocrine Center at Medical City, Dallas, TX, USA; Geralyn Spollett, Yale University School of Medicine, New Haven, CT, USA; Agathocles Tsatsoulis, University of Ioannina, Ioannina, Greece; Deborah Wexler, Massachusetts General Hospital, Boston, MA, USA; Bernard Zinman, LunenfeldTanenbaum Research Institute, University of Toronto and Mount Sinai Hospital, Toronto, ON, Canada. The final draft was also peer reviewed and approved by the Professional Practice Committee of the ADA and the Panel on Guidelines and Statements of the EASD.

Funding The face-to-face meeting was supported by the EASD. D. R. Matthews acknowledges support from the National Institute for Health Research.

Duality of interest During the past 12 months, the following relationships with companies whose products or services directly relate to the subject matter in this document are declared:

R. M. Bergenstal: membership of scientific advisory board, consultation services or clinical research support with
AstraZeneca, Boehringer Ingelheim, Eli Lilly, Merck \& Co., Novo Nordisk, Roche, Sanofi, and Takeda (all under contracts with his employer). Inherited stock in Merck \& Co. (previously held by family)

J. B. Buse: research and consulting with AstraZeneca; Boehringer Ingelheim; Bristol-Myers Squibb Company; Eli Lilly and Company; Johnson \& Johnson; Merck \& Co., Inc.; Novo Nordisk; Sanofi; and Takeda (all under contracts with his employer)

E. Ferrannini: membership on scientific advisory boards or speaking engagements for Merck Sharp \& Dohme, Boehringer Ingelheim, GlaxoSmithKline, Bristol-Myers Squibb/AstraZeneca, Eli Lilly \& Co., Novartis, and Sanofi. Research grant support from Eli Lilly \& Co., and Boehringer Ingelheim

S. E. Inzucchi: membership on scientific/research advisory boards for Boehringer Ingelheim, AstraZeneca, Intarcia, Lexicon, Merck \& Co., and Novo Nordisk. Research supplies to Yale University from Takeda. Participation in medical educational projects, for which unrestricted funding from Boehringer Ingelheim, Eli Lilly, and Merck \& Co. was received by Yale University

D. R. Matthews: has received advisory board consulting fees or honoraria from Novo Nordisk, GlaxoSmithKline, Novartis, Johnson \& Johnson, and Servier. He has research support from Johnson \& Johnson. He has lectured for Novo Nordisk, Servier, and Novartis

M. Nauck: research grants to his institution from Berlin-Chemie/ Menarini, Eli Lilly, Merck Sharp \& Dohme, Novartis, AstraZeneca, Boehringer Ingelheim, GlaxoSmithKline, Lilly Deutschland, and Novo Nordisk for participation in multicenter clinical trials. He has received consulting fees and/or honoraria for membership in advisory boards and/ or honoraria for speaking from Amylin, AstraZeneca, Berlin-Chemie/ Menarini, Boehringer Ingelheim, Bristol-Myers Squibb, Diartis Pharmaceuticals, Eli Lilly, F. Hoffmann-La Roche, GlaxoSmithKline, Hanmi, Intarcia Therapeutics, Janssen, Merck Sharp \& Dohme, Novartis, Novo Nordisk, Sanofi, Takeda, and Versartis, including reimbursement for travel expenses

A. L. Peters: has received lecturing fees and/or fees for ad hoc consulting from AstraZeneca, Bristol-Myers Squibb, Janssen, Eli Lilly, Novo Nordisk, Sanofi, and Takeda

A. Tsapas: has received research support (to his institution) from Novo Nordisk and Boehringer Ingelheim, and lecturing fees from Novartis, Eli Lilly and Boehringer Ingelheim

R. Wender: declares he has no duality of interest

Contribution statement All the named Writing Group authors contributed substantially to the document. All authors supplied detailed input and approved the final version. S. E. Inzucchi and D. R. Matthews directed, chaired and coordinated the input with multiple e-mail exchanges between all participants.

\section{Appendix}

The following scale was developed to categorise efficacy of the anti-hyperglycaemic drug classes, with data predominately based on placebo-controlled trials in monotherapy. The Writing Group acknowledges that this schema is somewhat arbitrary and that there are many different ways to assess the $\mathrm{HbA}_{1 \mathrm{c}}$-lowering effect of agents, including head-to-head trials. The results of all such trials are influenced by baseline $\mathrm{HbA}_{1 \mathrm{c}}$, drug type and dose, duration of treatment, wash-out from other anti-hyperglycaemic therapies, as well as adherence among participants to study medication and diet and 
exercise, among other factors. Accordingly, it remains challenging to evaluate and compare the 'potency' of antihyperglycaemic drugs. Moreover, mean differences between most agents, with some exceptions, are modest. Such data would be unlikely to reflect with any certainty the differential effect of a specific drug at a precise point in the treatment course in an individual patient.

\begin{tabular}{ll}
\hline Mean $\mathrm{HbA}_{\mathbf{1 c}}$ reduction & Efficacy category \\
Potential of $>2 \%(>22 \mathrm{mmol} / \mathrm{mol})$ & Very high \\
$>1-2 \%(>11-22 \mathrm{mmol} / \mathrm{mol})$ & High \\
$>0.5-1 \%(>5.5-11 \mathrm{mmol} / \mathrm{mol})$ & Intermediate \\
$\leq 0.5 \%(\leq 5.5 \mathrm{mmol} / \mathrm{mol})$ & Low \\
\hline
\end{tabular}

The following scale was developed to categorise cost of the anti-hyperglycaemic drug classes, using an online retail pharmacy tool for New Haven, Connecticut, in October 2014. We queried the lowest-priced member of each class at the highest prescribed dose for a 30-day supply. Insulin was assigned a 'variable' category, given the very wide range in cost, dependent on formulation and dose. The Writing Group acknowledges that this schema is also somewhat arbitrary but feels that it constitutes a reasonable valuation of healthcare expenditures. Costs are always of concern to health providers, though these may not be apparent to an individual patient covered by a health service, and may vary based on insurance coverage and other factors.

\begin{tabular}{ll}
\hline Daily cost in US\$ & Cost category \\
$<\$ 1$ & Low \\
$\$ 1-<\$ 2$ & Moderate \\
$\geq \$ 2$ & High \\
\hline
\end{tabular}

\section{References}

1. Inzucchi SE, Bergenstal RM, Buse JB et al (2012) Management of hyperglycemia in type 2 diabetes: a patient-centered approach: position Statement of the American Diabetes Association (ADA) and the European Association for the Study of Diabetes (EASD). Diabetes Care 35:1364-1379

2. Inzucchi SE, Bergenstal RM, Buse JB et al (2012) Management of hyperglycaemia in type 2 diabetes: a patient-centered approach. Position statement of the American Diabetes Association (ADA) and the European Association for the Study of Diabetes (EASD). Diabetologia 55:1577-1596

3. Stratton IM, Adler AI, Neil HA et al (2000) Association of glycaemia with macrovascular and microvascular complications of type 2 diabetes (UKPDS 35): prospective observational study. BMJ 321:405412

4. UKPDS Group (1998) Intensive blood-glucose control with sulphonylureas or insulin compared with conventional treatment and risk of complications in patients with type 2 diabetes (UKPDS
33). UK Prospective Diabetes Study (UKPDS) Group. Lancet 352: 837-853

5. Holman RR, Paul SK, Bethel MA, Matthews DR, Neil HA (2008) 10-year follow-up of intensive glucose control in type 2 diabetes. N Engl J Med 359:1577-1589

6. Gerstein HC, Miller ME, Byington RP et al (2008) Effects of intensive glucose lowering in type 2 diabetes. N Engl J Med 358:25452559

7. Vasilakou D, Karagiannis T, Athanasiadou E et al (2013) Sodium-glucose cotransporter 2 inhibitors for type 2 diabetes: a systematic review and meta-analysis. Ann Intern Med 159:262-274

8. Stenlöf K, Cefalu WT, Kim KA et al (2013) Efficacy and safety of canagliflozin monotherapy in subjects with type 2 diabetes mellitus inadequately controlled with diet and exercise. Diabetes Obes Metab 15:372-382

9. Schernthaner G, Gross JL, Rosenstock J et al (2013) Canagliflozin compared with sitagliptin for patients with type 2 diabetes who do not have adequate glycemic control with metformin plus sulfonylurea: a 52-week randomized trial. Diabetes Care 36:2508-2515

10. Roden M, Weng J, Eilbracht J et al (2013) Empagliflozin monotherapy with sitagliptin as an active comparator in patients with type 2 diabetes: a randomised, double-blind, placebo-controlled, phase 3 trial. Lancet Diabetes Endocrinol 1:208-219

11. Nauck MA, Del Prato S, Meier JJ et al (2011) Dapagliflozin versus glipizide as add-on therapy in patients with type 2 diabetes who have inadequate glycemic control with metformin: a randomized, 52-week, double-blind, active-controlled noninferiority trial. Diabetes Care 34:2015-2022

12. Cefalu WT, Leiter LA, Yoon KH et al (2013) Efficacy and safety of canagliflozin versus glimepiride in patients with type 2 diabetes inadequately controlled with metformin (CANTATA-SU): 52 week results from a randomised, double-blind, phase 3 non-inferiority trial. Lancet 382:941-950

13. Bakris GL, Fonseca VA, Sharma K, Wright EM (2009) Renal sodium-glucose transport: role in diabetes mellitus and potential clinical implications. Kidney Int 75:1272-1277

14. Ferrannini E, Solini A (2012) SGLT2 inhibition in diabetes mellitus: rationale and clinical prospects. Nat Rev Endocrinol $8: 495-502$

15. Rosenstock J, Seman LJ, Jelaska A et al (2013) Efficacy and safety of empagliflozin, a sodium glucose cotransporter 2 (SGLT2) inhibitor, as add-on to metformin in type 2 diabetes with mild hyperglycaemia. Diabetes Obes Metab 15:1154-1160

16. Chino Y, Samukawa Y, Sakai S et al (2014) SGLT2 inhibitor lowers serum uric acid through alteration of uric acid transport activity in renal tubule by increased glycosuria. Biopharm Drug Dispos 35:391404

17. Nyirjesy P, Sobel JD, Fung A et al (2014) Genital mycotic infections with canagliflozin, a sodium glucose co-transporter 2 inhibitor, in patients with type 2 diabetes mellitus: a pooled analysis of clinical studies. Curr Med Res Opin 30:1109-1119

18. Johnsson KM, Ptaszynska A, Schmitz B, Sugg J, Parikh SJ, List JF (2013) Vulvovaginitis and balanitis in patients with diabetes treated with dapagliflozin. J Diabetes Complicat 27: 479-484

19. Monami M, Nardini C, Mannucci E (2014) Efficacy and safety of sodium glucose co-transport-2 inhibitors in type 2 diabetes: a metaanalysis of randomized clinical trials. Diabetes Obes Metab 16:457466

20. Weir MR, Kline I, Xie J, Edwards R, Usiskin K (2014) Effect of canagliflozin on serum electrolytes in patients with type 2 diabetes in relation to estimated glomerular filtration rate (eGFR). Curr Med Res Opin 30:1759-1768

21. FDA briefing document (2013) Invokana (canagliflozin) tablets. [NDA 204042], US Food and Drug Administration 
22. Neal B, Perkovic V, de Zeeuw D et al (2013) Rationale, design, and baseline characteristics of the Canagliflozin Cardiovascular Assessment Study (CANVAS) - a randomized placebo-controlled trial. Am Heart J 166:217-223.e11

23. Balaji V, Seshiah V, Ashtalakshmi G, Ramanan SG, Janarthinakani M (2014) A retrospective study on finding correlation of pioglitazone and incidences of bladder cancer in the Indian population. Indian J Endocrinol Metab 18:425427

24. Kuo HW, Tiao MM, Ho SC, Yang CY (2014) Pioglitazone use and the risk of bladder cancer. Kaohsiung J Med Sci 30:94-97

25. Wei L, MacDonald TM, Mackenzie IS (2013) Pioglitazone and bladder cancer: a propensity score matched cohort study. Br J Clin Pharmacol 75:254-259

26. Dormandy JA, Charbonnel B, Eckland DJ et al (2005) Secondary prevention of macrovascular events in patients with type 2 diabetes in the PROactive Study (PROspective pioglitAzone Clinical Trial In macroVascular Events): a randomised controlled trial. Lancet 366: 1279-1289

27. Colhoun HM, Livingstone SJ, Looker HC et al (2012) Hospitalised hip fracture risk with rosiglitazone and pioglitazone use compared with other glucose-lowering drugs. Diabetologia 55:2929-2937

28. Scirica BM, Bhatt DL, Braunwald E et al (2013) Saxagliptin and cardiovascular outcomes in patients with type 2 diabetes mellitus. N Engl J Med 369:1317-1326

29. Scirica BM, Braunwald E, Raz I et al (2014) Heart failure, saxagliptin and diabetes mellitus: observations from the SAVOR - TIMI 53 randomized trial. Circulation 130:1579-1588

30. White WB, Cannon CP, Heller SR et al (2013) Alogliptin after acute coronary syndrome in patients with type 2 diabetes. N Engl J Med 369:1327-1335

31. White WB, Pratley R, Fleck P et al (2013) Cardiovascular safety of the dipetidyl peptidase-4 inhibitor alogliptin in type 2 diabetes mellitus. Diabetes Obes Metab 15:668-673

32. Egan AG, Blind E, Dunder K et al (2014) Pancreatic safety of incretin-based drugs - FDA and EMA assessment. N Engl J Med 370:794-797

33. Salpeter SR, Greyber E, Pasternak GA, Salpeter EE (2010) Risk of fatal and nonfatal lactic acidosis with metformin use in type 2 diabetes mellitus. Cochrane Database Syst Rev (4):CD002967. doi: 10.1002/14651858.CD002967.pub4

34. Lipska KJ, Bailey CJ, Inzucchi SE (2011) Use of metformin in the setting of mild-to-moderate renal insufficiency. Diabetes Care 34: 1431-1437

35. Nye HJ, Herrington WG (2011) Metformin: the safest hypoglycaemic agent in chronic kidney disease? Nephron Clin Pract 118: c380-c383

36. Pilmore HL (2010) Review: metformin: potential benefits and use in chronic kidney disease. Nephrology 15:412-418

37. Lu WR, Defilippi J, Braun A (2013) Unleash metformin: reconsideration of the contraindication in patients with renal impairment. Ann Pharmacother 47:1488-1497

38. National Institute for Health and Care Excellence (2009) Type 2 diabetes: the management of type 2 diabetes [CG87]. NICE, London

39. Groop PH, Del Prato S, Taskinen MR et al (2014) Linagliptin treatment in subjects with type 2 diabetes with and without mild-to-moderate renal impairment. Diabetes Obes Metab 16: $560-568$

40. Phung OJ, Sobieraj DM, Engel SS, Rajpathak SN (2014) Early combination therapy for the treatment of type 2 diabetes mellitus: systematic review and meta-analysis. Diabetes Obes Metab 16:410417

41. Musso G, Gambino R, Cassader M, Pagano G (2012) A novel approach to control hyperglycemia in type 2 diabetes: sodium glucose co-transport (SGLT) inhibitors: systematic review and metaanalysis of randomized trials. Ann Med 44:375-393
42. Goring S, Hawkins N, Wygant G et al (2014) Dapagliflozin compared with other oral anti-diabetes treatments when added to metformin monotherapy: a systematic review and network meta-analysis. Diabetes Obes Metab 16:433-442

43. Bosi E, Ellis GC, Wilson CA, Fleck PR (2011) Alogliptin as a third oral antidiabetic drug in patients with type 2 diabetes and inadequate glycaemic control on metformin and pioglitazone: a 52-week, randomized, double-blind, active-controlled, parallel-group study. Diabetes Obes Metab 13:1088-1096

44. Holman RR, Thorne KI, Farmer AJ et al (2007) Addition of biphasic, prandial, or basal insulin to oral therapy in type 2 diabetes. N Engl J Med 357:1716-1730

45. Eng C, Kramer CK, Zinman B, Retnakaran R (2014) Glucagon-like peptide-1 receptor agonist and basal insulin combination treatment for the management of type 2 diabetes: a systematic review and metaanalysis. Lancet. doi:10.1016/S0140-6736(14)61335-0

46. Diamant M, Nauck MA, Shaginian R et al (2014) Glucagon-like peptide 1 receptor agonist or bolus insulin with optimized basal insulin in type 2 diabetes. Diabetes Care 37:2763-2773

47. Buse JB, Bergenstal RM, Glass LC et al (2011) Use of twice-daily exenatide in basal insulin-treated patients with type 2 diabetes: a randomized, controlled trial. Ann Intern Med 154:103-112

48. Balena R, Hensley IE, Miller S, Barnett AH (2013) Combination therapy with GLP-1 receptor agonists and basal insulin: a systematic review of the literature. Diabetes Obes Metab 15:485-502

49. Charbonnel B, Bertolini M, Tinahones FJ, Domingo MP, Davies M (2014) Lixisenatide plus basal insulin in patients with type 2 diabetes mellitus: a meta-analysis. J Diabetes Complicat 28:880-886

50. Lane W, Weinrib S, Rappaport J, Hale C (2014) The effect of addition of liraglutide to high-dose intensive insulin therapy: a randomized prospective trial. Diabetes Obes Metab 16:827-832

51. Davidson MB, Raskin P, Tanenberg RJ, Vlajnic A, Hollander P (2011) A stepwise approach to insulin therapy in patients with type 2 diabetes mellitus and basal insulin treatment failure. Endocr Pract 17:395-403

52. Rosenstock J, Jelaska A, Frappin G et al (2014) Improved glucose control with weight loss, lower insulin doses, and no increased hypoglycemia with empagliflozin added to titrated multiple daily injections of insulin in obese inadequately controlled type 2 diabetes. Diabetes Care 37:1815-1823

53. Charbonnel B, DeFronzo R, Davidson J et al (2010) Pioglitazone use in combination with insulin in the prospective pioglitazone clinical trial in macrovascular events study (PROactive19). J Clin Endocrinol Metab 95:2163-2171

54. Shah PK, Mudaliar S, Chang AR et al (2011) Effects of intensive insulin therapy alone and in combination with pioglitazone on body weight, composition, distribution and liver fat content in patients with type 2 diabetes. Diabetes Obes Metab 13:505-510

55. Davidson MB, Navar MD, Echeverry D, Duran P (2010) U-500 regular insulin: clinical experience and pharmacokinetics in obese, severely insulin-resistant type 2 diabetic patients. Diabetes Care 33: 281-283

56. Hawa MI, Buchan AP, Ola T et al (2014) LADA and CARDS: a prospective study of clinical outcome in established adult-onset autoimmune diabetes. Diabetes Care 37:1643-1649

57. Zampetti S, Campagna G, Tiberti C et al (2014) High GADA titer increases the risk of insulin requirement in LADA: a 7-years of follow-up (NIRAD Study 7). Eur J Endocrinol 171:697-704

58. Nathan DM, Buse JB, Kahn SE et al (2013) Rationale and design of the glycemia reduction approaches in diabetes: a comparative effectiveness study (GRADE). Diabetes Care 36:2254-2261

59. Ismail-Beigi F, Moghissi E, Tiktin M, Hirsch IB, Inzucchi SE, Genuth S (2011) Individualizing glycemic targets in type 2 diabetes mellitus: implications of recent clinical trials. Ann Intern Med 154:554-559

60. Chiasson JL, Gomis R, Hanefeld M, Josse RG, Karasik A, Laakso M; STOP-NIDDM Trial Research Group (1998) The STOP-NIDDM 
Trial: an international study on the efficacy of an alpha-glucosidase inhibitor to prevent type 2 diabetes in a population with impaired glucose tolerance: rationale, design, and preliminary screening data. Diabetes Care 21:1720-1725

61. UKPDS Group (1998) Effect of intensive blood-glucose control with metformin on complications in overweight patients with type 2 diabetes (UKPDS 34). UK Prospect Diabetes Study (UKPDS) Group Lancet 352:854-865

62. Gaziano JM, Cincotta AH, O'Connor CM et al (2010) Randomized clinical trial of quick-release bromocriptine among patients with type 2 diabetes on overall safety and cardiovascular outcomes. Diabetes Care 33:1503-1508 